\title{
Prehospital Assessment of Non-Traumatic Abdominal Pain
}

\author{
Mahmoudreza Peyravi ${ }^{1,2,}$, Eric Carlström ${ }^{3,4}$, Per Örtenwal ${ }^{1}$ and Amir Khorram-Manesh ${ }^{1,4,5}$ \\ 1 Department of Surgery, Institute of Clinical Sciences, Sahlgrenska Academy, Gothenburg University, Gothenburg, Sweden \\ 2 Department of Health in Disasters and Emergencies, School of Management and Medical Informatics, Shiraz University of Medical Sciences, Shiraz, Iran \\ ${ }^{3}$ Health and Care Sciences, Sahlgrenska Academy, Gothenburg University, Gothenburg, Sweden \\ 4 University of South-Eastern Norway, Vestfold, Norway \\ ${ }^{5}$ Gothenburg Emergency Medicine Research Group, (GEMREG), Sahlgrenska Academy, Gothenburg University, Gothenburg, Sweden
}

* Corresponding author: Mahmoudreza Peyravi, Department of Health in Disasters and Emergencies, Health Human Resources Research Center, School of Management and Medical Informatics, Shiraz University of Medical Sciences, Shiraz, Iran. Tel: +987132340776; Email: peyravi110@gmail.com

Received 2021 March 15; Revised 2021 April 10; Accepted 2021 May 15.

\begin{abstract}
Background: Non-traumatic abdominal pain may have several causes and often needs physicians' consultation. In a pilot study, a template was used to assist ambulance nurses and technicians to assess this medical condition.

Objectives: This study aimed to evaluate the use of the template and the outcome of the pilot study.

Methods: A prehospital template (protocol) consisting of validated physiological and clinical scales, such as "Rapid Emergency Triage and Treatment Scale-Ambulances", "Patient Behavioral Pain Scale", and "Visual Analog Scale" were used to assist the ambulance crew in Shiraz, Iran, to assess all cases with non-traumatic abdominal pain. The outcome of the evaluation in this group was compared with a control group using the normal routine of assessment.

Results: Of 200 prehospital patients, 170 cases were eligible for evaluation. The majority of the patients in the protocol ( $\mathrm{n}=88$ ) and control groups $(n=82)$ were males $(n=46$ and $n=43$, respectively). There was a slight but statistically significant difference between the two groups in favor of the protocol group concerning the need for ambulance transport and direct admission to the hospital ( $<<0.02$ ).

Conclusion: Although prehospital assessment and management of non-traumatic abdominal pain continue to be challenging for emergency medical staff, educational initiatives together with prehospital guidelines may improve the accuracy of prehospital decisionmaking and open up for new fast-track diagnosis and direct admission to the hospital.
\end{abstract}

Keywords: Abdominal pain, Fast track, Prehospital care, Protocol, Triage

\section{Background}

Prehospital assessment of emergency patients offers an excellent opportunity to bring emergency departments (ED) closer to patients, unburden ED, and decrease the rates of mortality and morbidity by quick and proper management, including the use of ultrasonography, electrocardiography, and simple laboratory tests, and in some conditions, direct admission to the designated wards $(1,2)$. Such attempts have been successful for some groups of patients (e.g., patients with heart disease, hip fractures, and pediatric patients using prehospital protocols) (3-6).

Most common prehospital systems are either based on Anglo-American (staffed by paramedics), or Franco-German (staffed by physicians). A third version is also practiced in countries, such as Sweden with ambulances staffed with very skillful nurses (7). Although there is a higher accuracy of prehospital diagnosis by emergency physicians, compared to that of nurses or paramedics, studies have shown that the management of emergency patients by paramedics is both safe and possible (8-14). One way to increase the safety and accuracy of the paramedics' assessment is the use of guidelines and protocols (3-6).
Non-traumatic abdominal pain is a clinical challenge; however, its prehospital assessment and management may lead to a quicker admission of a patient to the right level of care. Studies evaluating prehospital abdominal pain are limited due to difficulties in differential diagnoses and the severity of the disease (15-17). However, retrospective comparison of non-traumatic abdominal pain diagnosis between a dispatch center and ED has shown that most of the patients diagnosed as having an emergency condition by the dispatch center do not have emergency conditions; in addition, the evaluation of ambulance crew on the scene is much closer to the definitive diagnosis at the hospital (15-17).

This study aimed at using a prehospital protocol to assess patients with non-traumatic abdominal pain in Shiraz, Iran. The Iranian Emergency Medical Services (EMS) was established in 1975 in close cooperation with American EMS. Since then, it has improved, expanded, and become a nationwide organization with the added responsibility to act during major incidents and disasters. Each ambulance is staffed with one nurse with expanded training in anesthesiology and one paramedic educated in Basic Life Support (six months training). Moreover, one ambulance is designed to work as a 
mobile Intensive Care Unit and is staffed with one general practitioner (GP), one nurse, and one paramedic. Another GP works as a consultant for paramedics at the dispatch center to make medical decisions in dubious cases. The response time, for a regular call, is set to less than eight min in cities and less than 15 min in suburban areas. However, this setting has not yet been achieved in larger urban areas (18).

\section{Objectives}

This study aimed to evaluate the use of the template and the outcome of the pilot study.

\section{Methods}

\subsection{Protocol Creation}

The authors searched, identified, and studied available publications dealing with the prehospital assessment of non-traumatic abdominal pain using a rapid evidence review technique (19). The prehospital protocol was developed based on the diagnostic pathway described in patients with acute abdominal pain, divided into emergency or nonemergency cases according to their severity, medical history, physical examination, and in some cases, laboratory parameters (20).

A reference group consisting of five senior healthcare professionals (a surgeon, an anesthesiologist, an emergency medicine physician, an ambulance nurse, and a dispatch nurse), were asked to evaluate the results of the search. They use the Nominal Group Technique (21) to discuss and suggest the structure of the protocol based on three criteria, including physiological characteristic of the abdominal pain, expressional characteristic of the patient, and duration of the abdominal pain using a combination of the following existing validated emergency protocols (Appendix 1).

\subsubsection{Rapid Emergency Triage and Treatment System- Ambulance (RETTS-A)}

A validated and regularly updated triage algorithm used in the prehospital setting in Sweden. It consists of different sections, including demographic characteristics (e.g., gender, age, and past medical history). Vital signs are evaluated systematically to categorize the patients into four triage groups (red, orange, yellow, and green) (Appendix 1). Red is the highest degree of emergency, followed by orange, yellow, and, green. The latter corresponds to patients who can wait for a more extended period for evaluation (22).

\subsubsection{Patient Behavioral Scale}

This scale depicts the severity of pain in a patient by explicitly looking at the patient's facial and body expression. It has five grades; however, the last category is only applicable in unconscious patients and was not used in this study (Appendix 1). The patient behavioral scale (PBS) is particularly important for patients who cannot express their pain, such as those with dementia. It is also essential to modify the pain in younger and pediatric patients (23-25).

\subsubsection{Visual Analog Scale}

The patients can express their pain within a range of 1-10 using the Visual Analog Scale (VAS). Moreover, they used VAS to show the severity of their abdominal pain in this study (Appendix 1) (26).

\subsubsection{Time Scale}

This scale was used based on the clinical experience of the expert group to indicate the duration of pain. More extended periods of pain indicated less severity, while the acute onset of pain would indicate an emergency $(20,26)$. The cut-off points for different priorities were chosen based on the consensus in the group of evaluators and after adjustment of the template.

\subsection{Protocol utilization}

The medical and academic expert group outlined five fictive medical conditions based on real cases and adjusted to mirror a broad set of patients to adjust protocol's dimensions to satisfy all members with its feasibility, relevance, comprehensiveness, clarity, logic, and accuracy. These cases were ruptured aorta aneurysm, perforated sigmoid diverticulitis, appendicitis, gastroenteritis, and non-specific abdominal pain.

All ambulance crews were educated to understand and utilize the protocol. The evaluation started with Rapid Emergency Triage and Treatment System-Ambulance (RETTS-A). The patients falling into red or orange categories were emergency cases and were transported to the hospital immediately. Those categorized into yellow or green continued to the next step and were evaluated by the PBS and VAS. The points obtained from the time scale were added to the sum of PBS and VAS. Following that, the patients triaged as red or orange based on the cut-off points were transported to the hospital, while the remaining patients were recommended to book a visit at their healthcare center (Appendix 1).

An emergency nurse at the dispatch center, who was not involved in the study, prospectively randomized the patients into the Protocol Group (PG) and Control Group (CG). Those included in the PG were assessed according to the protocol and those included in CG were handled routinely. Contact with the physician at the dispatch center was only made if the status deteriorated or it was not possible to follow the protocol. All included patients were followed up after one month. 


\subsection{Study Population}

\subsubsection{Venue and time}

This study was carried out from May 2015 to May 2016 in Shiraz, Iran. Data analysis and evaluation of the study were conducted in 2017. Shiraz is located in the south of Iran and is the capital of Fars province with a land area of $225-\mathrm{km}^{2}$ and 1.7 million inhabitants.

\subsubsection{Patients}

The inclusion criteria were: 1) patients with nontraumatic abdominal pain, who were registered at the dispatch center's registry and for whom an ambulance was sent, and 2) the presence of RETTS-A inclusion criteria (i.e., patients between 16 and 65 years old) with no registered significant past medical history (e.g., cardiovascular diseases, neurologic and psychiatric disorders, history of surgery, pregnancy, or liver diseases). On the other hand, the patients under 16 and over 65 years of age or with a significant past medical history were excluded from the study.

All included patients consented to follow-up. A 30day follow-up questionnaire was sent out to evaluate patients' condition and possible re-admission, if dismissed from the scene as well as their clinical diagnosis, treatment, and response to therapy, if readmitted (Appendix 2). The patients referred to a GP were followed up by phone. Moreover, those admitted to the hospital were followed up using their medical records and final diagnosis when discharged. In addition, those, who were admitted, treated with medication for more than three days, underwent surgery, or were subjected to any other intervention only available at the hospital were classified as in need of ambulances. Others were classified as not needing ambulances.

\subsubsection{Outcomes, exposures, predictors, confounding factors, and effect modifiers}

The outcome was complete assessment decisions on the diagnosis after the ED visit or when leaving after hospitalization. The Ethics Committee of Shiraz University of Medical Sciences, Shiraz, Iran, regarded potential patient exposure in relation to possible gains from the study. Informed consent was practiced during the study. The portion of confounding factors was unknown since no regression or correlation analyses were performed; however, potential confounding factors were identified as varying knowledge on the assessment technique between ambulance teams and varying priorities due to the density of ambulances. Predictors include three factors, namely PBS, VAS, and Time scale. The effect modifiers are the knowledge and skill of the technicians that eliminate possible bias.

\subsection{Statistical Analysis}

The sample size of this study was calculated considering a significance level $(\alpha)=5 \%$, power $=$ $90 \%$, standard deviation of outcome $=10$, and noninferiority limit of $d=5$. The result indicated a required sample size of 69 patients per group and a total sample size of 138 . Due to the sample attrition, 100 patients were assigned into each group; therefore, the overall sample size reached 200 patients. Data analysis was carried out in SPSS software (version 19; SPSS Inc., Chicago, Illinois, USA). The mean and standard deviation (SD) were used for descriptive statistical analysis. Moreover, sensitivity, specificity, and predictive values were also calculated, and the confidence interval was measured by Stata software (version 12) using the Weld method.

\subsection{Ethical considerations}

The Ethics Committee of Shiraz University of Medical Sciences, Shiraz, Iran, approved the study protocol (2011-100). Initially, an ambulance crew contacted the informed patients or their relatives (if applicable, it was conducted orally). They were also told that they would be contacted for followup. At follow-up, the patients or their families were again informed before all follow-up questions were asked.

\section{Results}

\subsection{Fictive patients}

A small group of nurses and paramedics used the protocol and assessed fictive patients. The patients with ruptured aorta aneurysm and perforated diverticulitis were captured on RETTS-A. Of the three remaining patients, those with appendicitis and gastroenteritis were doomed to transport according to the outcomes obtained from PBS and VAS scales, and the last patient was recommended a GP visit.

\subsection{Prehospital patients with non-traumatic abdominal pain}

4.2.1. Demographic characteristics and diagnosis

No protocol violations were reported in this study. Of 200 randomized patients, 12 and 18 patients from PG and CG were excluded, respectively, due to RETTS-A's exclusion criteria (age or previous medical history). The final numbers of the patients were then 88 and 82 in the PG and CG, respectively. The PG consisted of $46(52.3 \%)$ male and $42(47.7 \%)$ female patients with a mean age of $40.25 \pm 14.05$. On the other hand, there were $43(52.4 \%)$ male and 39 $(47.6 \%)$ female patients in the CG with a mean age of $37.87 \pm 13.94$ years. Table 1 shows the final diagnosis after clinical evaluation and admission to the hospital in the PG and CG. All diagnoses were taken from the medical files. Surgical diseases were the most common diagnosis in both groups. Other clinical diagnoses were internal medicine and gynecological diseases, infections, and poisoning. 


\begin{tabular}{|c|c|c|}
\hline \multirow{2}{*}{ Clinical Diagnosis } & \multicolumn{2}{|c|}{ Frequency (\%) } \\
\hline & Protocol Group & Control Group \\
\hline Surgical diseases & $39(44.3)$ & $24(29.3)$ \\
\hline Internal medicine diseases & $23(26.1)$ & $19(23.2)$ \\
\hline Poisoning & $8(9.1)$ & $3(3.7)$ \\
\hline Unknown & $7(8)$ & 17 (20.7) \\
\hline Gynecological diseases & $5(5.7)$ & $7(8.5)$ \\
\hline Psychiatric disorder & $4(4.5)$ & $4(4.9)$ \\
\hline Infection & $2(2.3)$ & $8(9.8)$ \\
\hline
\end{tabular}

\subsubsection{Needs for transportation to the hospital}

In total, 84 (95.5\%) PG patients were transported to the hospital by ambulance, and four patients were recommended to visit their GP. All 84 patients passed the RETTS-A evaluation scale.

The decision made to transport these patients was based on the sum of other scales in the protocol. In the CG, the EMS staff transported 71 (86.5\%) patients to the hospital by ambulance after consultation with a physician, and the remaining 11 patients were recommended to visit a GP. Figure 1 shows the whole process of inclusion, exclusion, and outcome in both groups of transported and not transported patients.

\subsubsection{Hospital admission and outcome}

All patients transported to the hospital in both groups were examined and admitted if needed. Table 2 tabulates the assessment and treatment process at the hospitals.
In the PG, 70 cases out of 84 patients (83.3\%) were admitted and thus classified as needing an ambulance based on the criteria mentioned under the method section (admitted for treatment and surgery or died due to the worsening of their conditions). Totally, three deaths in this group occurred in patients with liver cirrhosis $(n=2)$ and abdominal cancer ( $\mathrm{n}=1$; not previously known). The rest of the patients in this group $(n=14)$ were discharged from the ED. In the CG, the number of admitted and treated patients in need of ambulance transportation was 49 cases out of 71 patients $(69.0 \%)$. The remaining 22 patients were discharged. The number of patients with abdominal pain leading to an intervention or treatment was higher in $\mathrm{PG}$, compared to the $\mathrm{CG}$ (70 vs. 49).

\subsubsection{Follow-up}

Table 3 summarizes the follow-up results in both transported and not transported patients. Of those

Protocol Group (PG)

Control Group (CG)

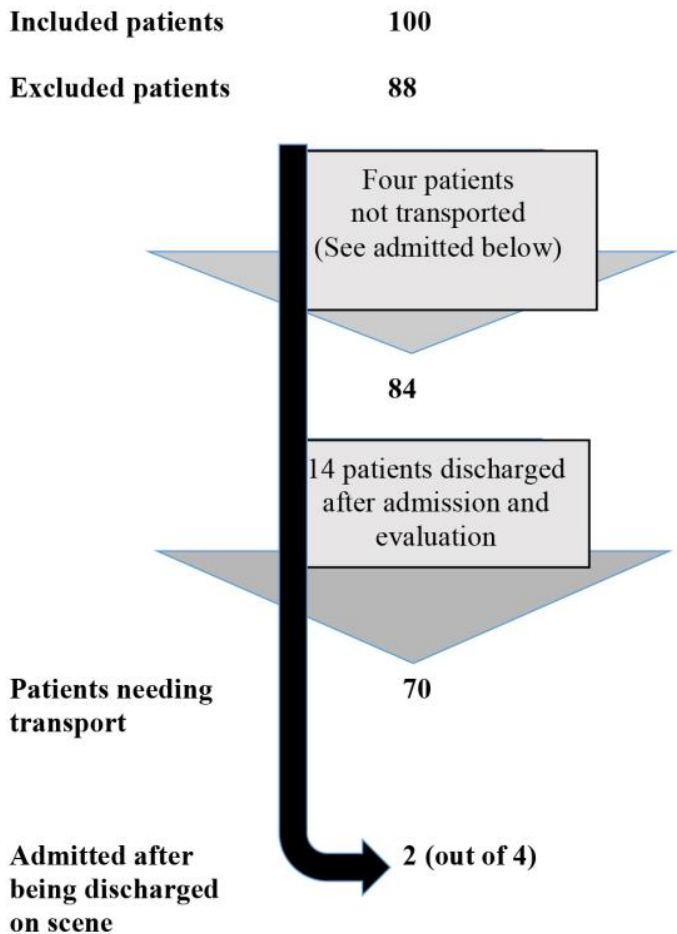

100

82

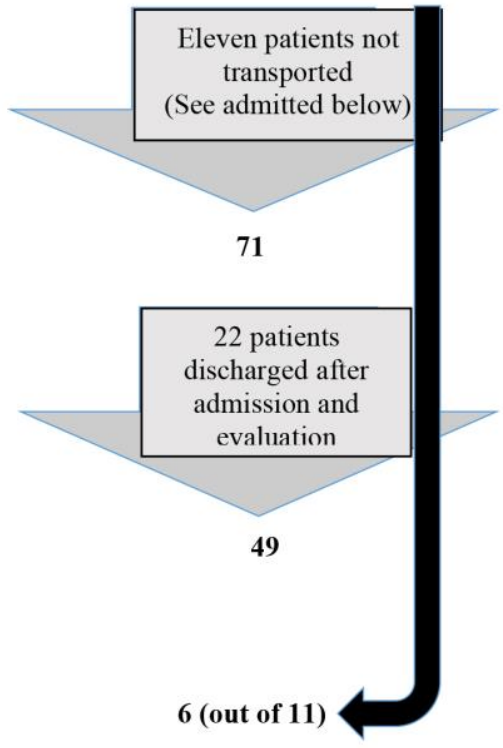

Figure 1. An overview of the process of inclusion and outcome in groups A \& B 


\begin{tabular}{lcc}
\hline \multicolumn{2}{l}{ Table 2. Outcome of the patients in both groups based on the admission process, treatments, and procedures given } \\
\hline Outcome & $\begin{array}{c}\text { Protocol Group } \\
\text { Number (Frequency \%) }\end{array}$ & $\begin{array}{c}\text { Control Group } \\
\text { Number (Frequency \%) }\end{array}$ \\
\hline Total discharged & $14(16.6 \%)$ & $22(31 \%)$ \\
Total admitted & $70(83.4 \%)$ & $49(69 \%)$ \\
& $53(63.1 \%)$ & $38(53.5 \%)$ \\
1. Admission, medication, and treatment & $13(15.5 \%)$ & $11(15.5 \%)$ \\
2. Admission with operation & $3(3.6 \%)$ & 0 \\
3. Deaths & $1(1.2 \%)$ & 0 \\
Total transported & $84(100 \%)$ & $71(100 \%)$ \\
\end{tabular}

not transported to the hospital in PG $(n=4)$, two patients were admitted to the hospital within the follow-up time (50\%), while in the CG, six out of 11 patients were admitted to the hospital (54\%). The difference between the two groups concerning the accuracy of the true transporting cases with nontraumatic abdominal pain was statistically significant. Based on the test of equal proportions, there is a significant difference between the two groups regarding the prevalence of needing an ambulance $\left(P_{A}=0.82, P_{B}=0.67\right.$, Sig $\left.=0.02\right) \quad$ (Table 3). The predictive validity of the protocol was measured by the sensitivity and specificity of the diagnosis using the protocol, compared to that of the CG (Table 3, 4). The decision made by the EMS staff to transport the patient was defined as a positive result. If the patient transported by the ambulance was proven to be significantly sick in the follow-up, the case was then considered truly positive; otherwise, it was a falsepositive case. True negative cases were defined as those who were not transported and proven not to be sick; otherwise, they would be false-negative cases. The protocol showed sufficient sensitivity; however, the specificity was somewhat weaker.

Half of the "not transported" patients (Two patients out of four in PG and six out of eleven in CG) were re-admitted to the hospital during the follow-up period. However, there was no statistically significant difference between $P G$ and CG regarding the number of re-admissions.

\begin{tabular}{lcc}
\hline $\begin{array}{l}\text { Table 3. Number of patients in need of ambulance based on the criteria mentioned under the method section. The difference } \\
\text { between the two groups was statistically significant }(\mathrm{P}<0.02)\end{array}$ & in favor of the PG group \\
\hline Protocol Group & Needing an Ambulance & Not-needing an Ambulance \\
\hline Transported & 2 & 14 \\
Not transported & Needing an ambulance & 2 \\
Control group & 49 & Not-needing an ambulance \\
Transported & 6 & 22 \\
Not transported & 6 & 5 \\
\hline
\end{tabular}

Table 4: Sensitivity, specificity, and predictive value of the diagnosis using protocol or no protocol

\begin{tabular}{lccc}
\hline Zone & Protocol Group & Control Group & Ratio Group A/B \\
\hline Sensitivity & $0.97(0.93-1)$ & $0.89(0.80-0.97)$ & 1.08 \\
Specificity & $0.12(0-0.28)$ & $0.18(0.03-0.33)$ & 0.69 \\
Positive predictive value & $0.83(0.71-0.83)$ & $0.69(0.58-0.79)$ & 1.2 \\
Negative predictive value & $0.25(0.01-0.98)$ & $0.45(016-0.74)$ & 0.55 \\
\hline
\end{tabular}

\section{Discussion}

The vast majority of the patients in both groups were transported to the hospital. However, of those admitted to the hospital, the number of true cases (abdominal pain) was more prominent in the protocol group, compared to the control group $(n=70$; $83.4 \%$ vs. $\mathrm{n}=49 ; 69 \%)$. There was a slight but statistically significant difference in the accuracy of the decision made by EMS staff, who used the protocol $(\mathrm{P}<0.02)$. This result is in accordance with the findings of the

9earlier studies showing that protocols could replace radio contact with dispatch physicians $(7,27,28)$.

Non-traumatic abdominal pain is a clinical challenge since a wide range of diseases from minor to potentially deadly conditions can cause it. In this study, a new protocol was tested on some ordinary cases in an ED. In our prehospital group of patients, the causes of non-traumatic abdominal pain varied from surgical diseases to poisoning. In many cases, the cause of the pain was unknown. Lammer et al. compared six protocols for non-traumatic abdominal pain and revealed no significant difference among these six protocols in terms of helping EMS staff with on-scene decision-making (16). Nevertheless, the protocols used at that time were less detailed and non-specific. The possibility of monitoring vital parameters using new technologies may improve modern protocols. In opposite to the previous protocols, the current 
protocol uses various validated algorithms, such as RETTS-A triage, PBS, and VAS, which have all been used in clinical practice (20).

The ED overcrowding is a serious issue for patient safety (1, 2). Digestive symptoms, including abdominal pain, constitute around $11 \%$ of the visits among patients presenting by ambulance with moderate or severe pain $(20,29)$. These cases represent a large number of patients, who do not need to wait at ED and can be transported directly to the appropriate ward and the required level of care. Transporting the right patient by ambulance to the right medical facility is a significant challenge for the EMS staff (17, 30). In a Franco-German EMS, physicians are actively working with the patients, and a decision can be made on the scene based on their medical experience and the patient's medical condition. In Anglo-American EMS, the staff needs to consult with a physician in doubtful cases (17-18). However, the medical decisions made by the physicians in charge depend on their knowledge, experience, and the accuracy of the information (physiology and anatomy) they receive from the ambulance crew. In some parts of the world, paramedics are used as first-line healthcare providers equipped with standardized information, protocols, and guidelines to evaluate the patient's vital signs and strengthen the process of decisionmaking (30-32). Previous studies have shown that targeted guidelines for specific conditions decrease mortality and morbidity, followed by an increase in patient safety (33-36). These guidelines may also be used to transfer some medical conditions directly to the wards in hospitals as fast track (3-6).

\section{Limitations}

Regarding the limitations of this study, one can refer to the limited publications on prehospital guidelines and diagnostic protocols in non-traumatic abdominal pain, which does not allow any comparison with earlier studies. Furthermore, in this study, the patients' demographic characteristics were matched to minimize any biases. Age and gender confounders, as well as the underlying diseases, were also considered. The sensitivity of the study was high, which indicates the validity of the used protocol, compared to the control group in diagnosing the condition. However, larger cohort and multi-centric studies are needed for further generalization of the tool.

Another limitation might be the random selection of the patients vs. the EMS staff. Since the number of ambulances and staff is limited, there is an obvious risk that one EMS personnel receives both categories of patients. However, by letting the randomization be performed by a nurse at the dispatch center, the risk for any influence on the use of the protocol on the routinely managed cases must be minimized. In addition, the staff was trained thoroughly to eliminate possible errors or biases. Another limitation might be the design of the protocol, the points given for each section, and the final cut-off points for decision-making. However, they were all a product of evaluation by five senior health professionals, evaluation of fictive cases, a literature study, and evaluators' experience. Finally, despite the adequate number of patients included in this study, our findings should be subject to further evaluation and new randomized studies.

\section{Conclusion}

Although no template substitutes a physician's knowledge and ability in decision-making in clinical cases, such as non-traumatic abdominal pain, it might increase the safety and accuracy of the EMS decision regarding the transportation to the hospital and direct admission to the responsible ward when physicians are not available. Such a prehospital template may also facilitate a new fast-track diagnosis and decreases the burden of ED overcrowding.

\section{Acknowledgment}

None

\section{Footnotes}

Authors' Contributions: All authors contributed equally in designing, evaluating, discussing, writing, and proofreading the manuscript; moreover, they approved the final submitted version.

Conflict of Interest: The authors declare no conflict of interest.

Ethical Approval: The Ethics Committee of Shiraz University of Medical Sciences, Shiraz, Iran, approved the study protocol (2011-100).

Funding/Support: Nil.

Financial Disclosure: None declared.

Informed consent: Informed consent was obtained from all individual participants included in the study.

\section{References}

1. Di Somma S, Paladino L, Vaughan L, Lalle I, Magrini L, Magnanti M. Overcrowding in emergency department: an international issue. Intern Emerg Med. 2015;10(2):171-5. doi: 10.1007/ s11739-014-1154-8. [PubMed: 25446540].

2. Khorram-Manesh A, Wennman I, Andersson B, Dahlén Holmqvist L, Carlson T, Carlström E. Reasons for longer LOS at the emergency departments: practical, patient-centred, medical, or cultural? Int J Health Plann Manage. 2019; 34(4):e1586-96. doi: 10.1002/hpm.2849. [PubMed: 31271229].

3. Steg PG, Cambou JP, Goldstein P, Durand E, Sauval P, Kadri Z, et al. Bypassing the emergency room reduces delays and mortality in ST elevation myocardial infarction: the USIC 2000 registry. Heart. 2006;92(10):1378-83. doi: 10.1136/hrt.2006.101972. [PubMed: 16914481]

4. Doggen CJ, Zwerink M, Droste HM, Brouwers PJ, van Houwelingen GK, van Eenennaam FL, et al. Prehospital paths 
and hospital arrival time of patients with acute coronary syndrome or stroke, a prospective observational study. BMC Emerg Med. 2016;16:3. doi: 10.1186/s12873-015-0065-y. [PubMed: 26748628].

5. Leyenaar JK, O'Brien ER, Malkani N, Lagu T, Lindenauer PK. Direct admission to hospital: a mixed methods survey of pediatric practices, benefits, and challenges. Acad Pediatr. 2016;16(2):175-82. doi: 10.1016/j.acap.2015.07.002. [PubMed: 26293551].

6. Gromov K, Willendrup F, Palm H, Troelsen A, Husted H. Fasttrack pathway for reduction of disclosed hip arthroplasty reduces surgical delay and length of stay. Acta Orthop. 2015;88(3):335-8. doi: 10.3109/17453674.2015.1007416. [PubMed: 25619606].

7. Haner A, Örninge P, Khorram-Manesh A. The role of physicianstaffed ambulances: the outcome of a pilot study. J Acute Dis. 2015;4(1):63-7. doi: 10.1016/S2221-6189(14)60086-X.

8. Finn JC, Fatovich DM, Arendts G, Mountain D, Tohira H, Williams TA, et al. Evidence-based paramedic models of care to reduce unnecessary emergency department attendance-feasibility and safety. BMC Emerg Med. 2013;13:13. doi: 10.1186/1471-227X-13-13. [PubMed: 23855265].

9. Hoyle S, Swain AH, Fake P, Larsen PD. Introduction of an extended care paramedic model in New Zealand. Emerg Med Australas. 2012;24(6):652-6. doi: 10.1111/j.17426723.2012.01608.x. [PubMed: 23216727].

10. Grantham H, Hein C, Elliott R. South Australian ambulance service (SAAS) extended care paramedic (ECP) pilot project. $J$ Emerg Primary Health Care. 2010;8(3):32.

11. Heuer JF, Gruschka D, Crozier TA, Bleckmann A, Plock E, Moerer 0 , et al. Accuracy of prehospital diagnoses by emergency physicians: comparison with discharge diagnosis. Eur J Emerg Med. 2012;19(5):292-6. doi: 10.1097/MEJ. 0b013e32834ce104. [PubMed: 21971293].

12. Christie A, Costa-Scorse B, Nicholls M, Jones P, Howie G. Accuracy of working diagnosis for patients presenting with dyspnea. Emerg Med Australas. 2016;28(5):525-30. doi: 10.1111/1742-6723.12618.

13. Williams TA, Blacker D, Arendts G, Patrick E, Brink D, Finn J. Accuracy of stroke identification by paramedics in a metropolitan prehospital setting: a cohort study. Australas J Paramed. 2017;14(2):1-10. doi: 10.33151/ajp.14.2.521.

14. Silvestri S, Rothrock SG, Kennedy D, Ladde J, Bryant M, Pagane J. Can paramedics accurately identify patients who do not require emergency department care? Prehosp Emerg Care. 2002;6(4):387-90. doi: 10.1080/10903120290937987. [PubMed: 12385603].

15. Viniol A, Keunecke C, Biroga T, Stadje R, Dornieden K, Bösner S, et al. Studies of the symptom abdominal pain--a systematic review and meta-analysis. Fam Pract. 2014;31(5):517-29. doi: 10.1093/fampra/cmu036. [PubMed: 24987023].

16. Lammers RL, Roth BA, Utecht T. Comparison of ambulance dispatch protocols for nontraumatic abdominal pain. Ann Emerg Med. 1995;26(5):579-89. doi: 10.1016/s0196-0644(95) 70008-0. [PubMed: 7486366].

17. Khorram-Manesh A, Montán KL, Hedelin A, Kihlgren M, Örtenwall P. Prehospital triage, the discrepancy in prioritysetting between the emergency medical dispatch center and ambulance crews. Eur J Trauma Emerg Surg. 2011;37(1):73-8. doi: 10.1007/s00068-010-0022-0. [PubMed: 26814754]

18. Peyravi M, Örtenwal P, Djalali A, Khorram-Manesh A. An overview of Shiraz emergency medical services, dispatch to treatment. Iran Red Crescent Med J. 2013;15(9):823-8. doi: 10.5812/ircmj.10982. [PubMed: 24616794].

19. Haby MM, Chapman E, Clark R, Barreto J, Reveiz L, Lavis JN. What are the best methodologies for rapid reviews of the research evidence for evidence-informed decision making in health policy and practice: a rapid review. Health Res Policy Syst. 2016;14(1):83. doi: 10.1186/s12961-016-0155-7. [PubMed: 27884208].

20. Gans SL, Pols MA, Stoker J, Boermeester MA, Expert Steering Group. Guideline for the diagnostic pathway in patients with acute abdominal pain. Dig Surg. 2015;32(1):23-31. doi:
$10.1159 / 000371583$

21. McMillam SS, King M, Tully MP. How to use the nominal group and Delphi techniques. Int J Clin Pharm. 2016;38(3):655-62. doi: 10.1007/s11096-016-0257-x. [PubMed: 26846316].

22. Mirhaghi A, Christ M. Revision for the rapid emergency triage and treatment system adult (RETTS-A) needed? Scand J Trauma Resusc Emerg Med. 2016;24(1):55. doi: 10.1186/ s13049-016-0254-z. [PubMed: 27118551]

23. Hylén M, Akerman E, Alm-Roijer C, Idvall E. Behavioral Pain Scale-translation, reliability, and validity in a Swedish context. Acta Anaesthesiol Scand. 2016;60(6):821-8. doi: 10.1111/aas.12688. [PubMed: 27251598].

24. Kahl C, Cleland JA. Visual analogue scale, numeric pain rating scale and the McGill Pain Questionnaire: an overview of psychometric properties. Phys Ther Rev. 2005;10(2):123-8. doi: $10.1179 / 108331905 X 55776$.

25. Herr K, Coyne PJ, Key T, Manworren R, McCaffery M, Merkel S, et al. Pain assessment in the nonverbal patient: position statement with clinical practice recommendations. Pain Manag Nurs. 2006;7(2):44-52. doi: 10.1016/j.pmn.2006.02.003.

26. Ariely D. Combining experiences over time: the effects of duration, intensity changes and on-line measurement on retrospective pain evaluations. J Behav Dec Mak. 1998; 11(1):19-45. doi: 10.1002/(SICI)1099-0771(199803)11:1< 19::AID-BDM277>3.0.CO;2-B.

27. Hoffman J, Luo J, Schriger DL, Silver L. Does paramedic-base hospital contact result in beneficial deviations from standard prehospital protocols? West J Med. 1990;153(3):283-7. [PubMed: 2219893]

28. Sullivan F, Williams KA. Physician medical direction of emergency medical services. R I Med J. 2013;96(12):28-30. [PubMed: 24303514].

29. McLean SA, Maio RF, Domeier RM. The epidemiology of pain in the prehospital setting. Prehosp Emerg Care. 2002;6(4):402-5. doi: 10.1080/10903120290938021. [PubMed: 12385606].

30. Peyravi M, Örtenwall P, Khorram-Manesh A. Can medical decision-making at the scene by EMS staff reduce the number of unnecessary ambulance transportations, but still be safe? PLoS Curr. 2015;7:1-9. doi: 10.1371/currents.dis. f426e7108516af698c8debf18810aa0a. [PubMed: 26203394].

31. Merz TM, Etter R, Mende L, Barthelmes D, Wiegand J, Martinolli L, et al. Risk assessment in the first fifteen minutes: a prospective cohort study of a simple physiological scoring system in the emergency department. Crit Care. 2011; 15(1):R25. doi: 10.1186/cc9972. [PubMed: 21244659].

32. Lamantia MA, Stewart PW, Platts-Mills TF, Biese KJ, Forbach C, Zamora E, et al. Predictive value of initial triage vital signs for critically ill older adults. West J Emerg Med. 2013;14(5):453-60. doi: 10.5811/westjem.2013.5.13411. [PubMed: 24106542].

33. Charpentier S, Sagnes-Raffy C, Cournot M, Cambou JP, Ducassé $\mathrm{JL}$, Lauque D, et al. Determinants and prognostic impact of compliance with guidelines in reperfusion therapy for STsegment elevation myocardial infarction: results from the ESTIM Midi-Pyrénées Area. Arch Cardiovasc Dis. 2009; 102(5):387-96. doi: 10.1016/j.acvd.2009.02.011. [PubMed: 19520324].

34. Kirves H, Skrifvars MB, Vähäkuopus M, Ekström K, Martikainen $\mathrm{M}$, Castren M. Adherence to resuscitation guidelines during prehospital care of cardiac arrest patients. Eur J Emerg Med. 2007;14(2):75-81. doi: 10.1097/MEJ.0b013e328013f88c. [PubMed: 17496680].

35. Ljunggren M, Castrén M, Nordberg M, Kurland L. The association between vital signs and mortality in a retrospective cohort study of an unselected emergency department population. Scand J Trauma Resusc Emerg Med. 2016;24:21. doi: 10.1186/s13049-016-0213-8. [PubMed: 26940235]

36. Doherty SR, Jones PD, Davis L, Ryan NJ, Treeve V. Evidence-based implementation of adult asthma guidelines in the emergency department: a controlled trial. Emerg Med Australas. 2007;19(1):31-8. doi: 10.1111/j.1742-6723.2006. 00910.x. [PubMed: 17305658]. 
Prehospital support for decision-making in non-traumatic abdominal pain (modified RETTS ${ }^{\mathrm{TM}}$ )

PKMC

Ambulance No.:

Cause of contact:

Time of arrival:

Date:

Patient No.

Current problem:

\begin{tabular}{|c|c|c|c|c|c|c|c|c|c|c|c|}
\hline & YES & NO & & YES & NO & & YES & NO & & YES & NO \\
\hline Healthy & & & Cardiac insufficiency & & & Bleeding tendency & & & Immunosuppression & & \\
\hline Cardiovascular disease & & & cerebrovascular disease & & & Respiratory disease & & & Hypertension & & \\
\hline Kidney disease & & & Liver disease & & & Malignancy & & & Diabetes, tablets & & \\
\hline Diabetes, Insulin & & & Allergic & & & operated $<3$ months & & & & & \\
\hline
\end{tabular}

Part 1: a) PHYSIOLOGIC EVALUATION/TRIAGE

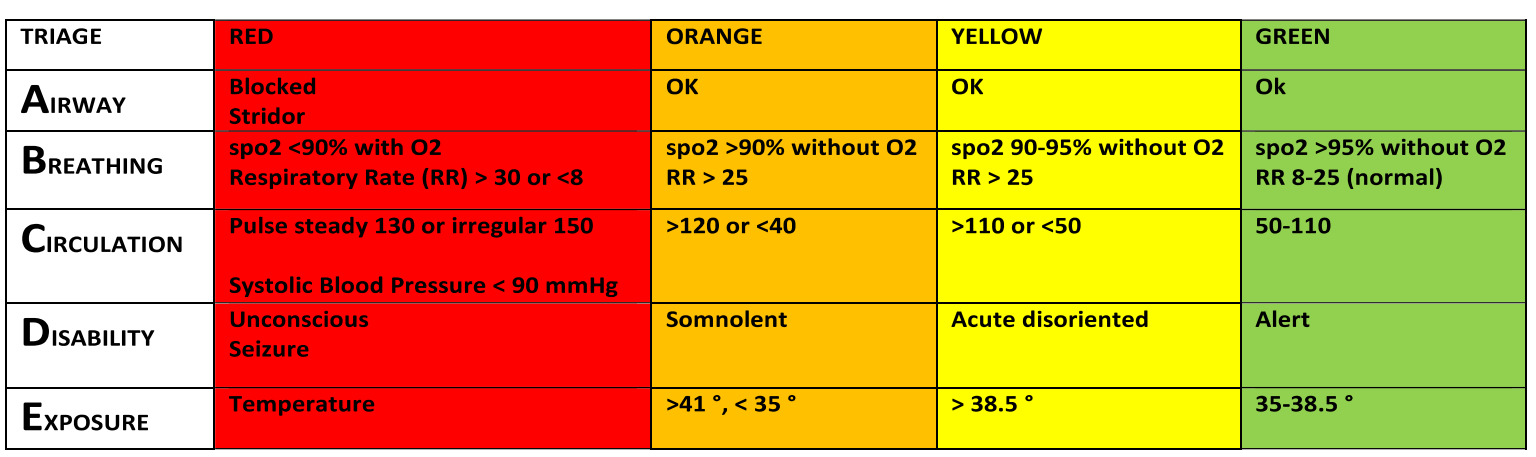

Part 2: OBJECTIVE EVALUATION OF ABDOMINAL PAIN

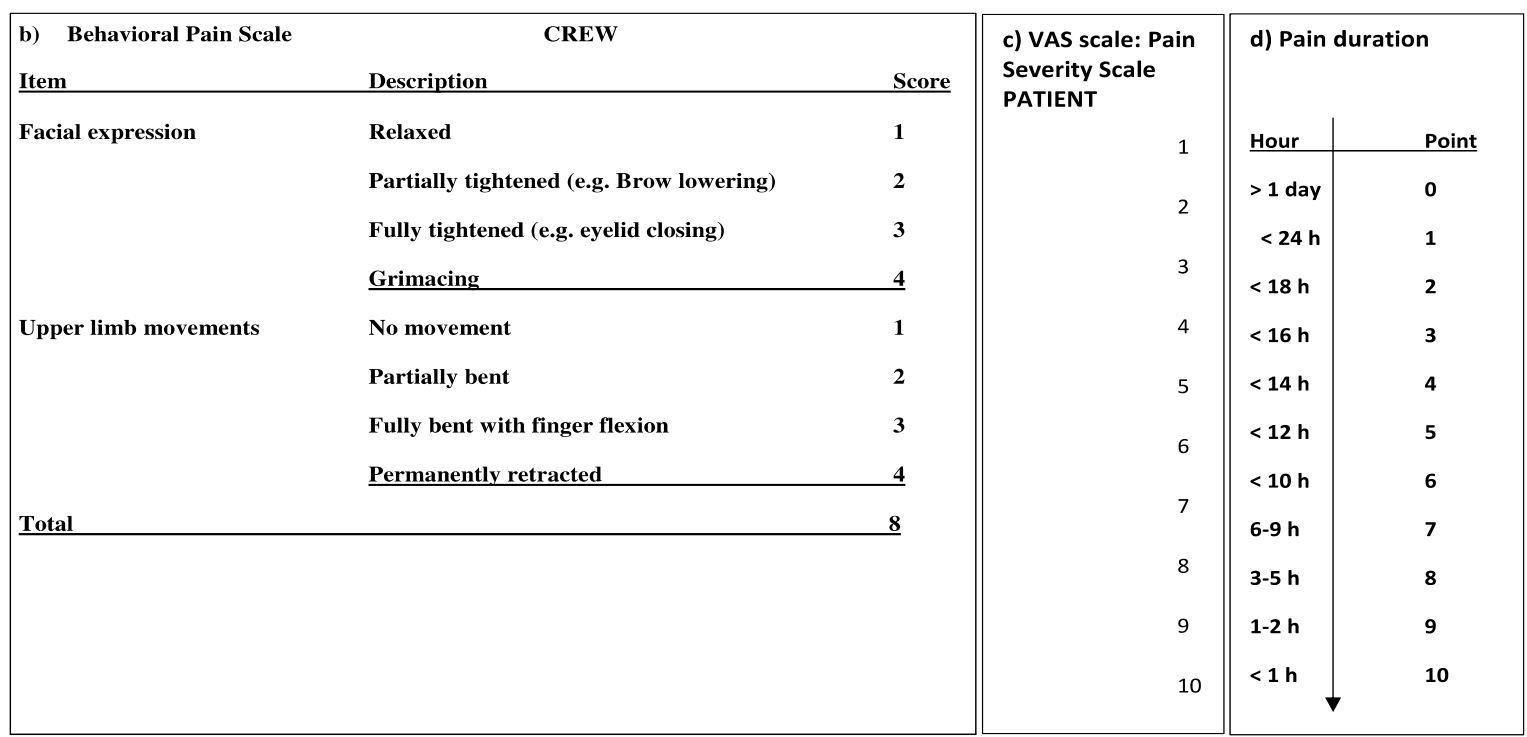

AVERAGE POINT "VAS" PATIENTS AND CREW + POINT OBTAINED PAIN DURATION

\begin{tabular}{|c|c|c|c|c|c|c|c|c|}
\hline$b+c+d$ & 23-28 Points & RED & 16-22 Points & ORANGE & 11-15 Points & YELLOW & $<10$ Points & GREEN \\
\hline
\end{tabular}

Appendix 1. 
Follow-up questionnaire in non-traumatic abdominal pain.

Patient consent to follow-up No
Patient name:
Recent admission to the hospital:
Questions to be asked or answered by phone/written;
1. What were the main reasons to request an ambulance and your chief
complaint?
2. What were your symptoms?
3. Was the decision not to be transported to the hospital your own, or did the
ambulance crew make it?
4. If it was the staff's decision, were you satisfied with it?
5. Did your symptoms continue after being discharged? If your answer is yes,
what were those?
6. Wid you visit a clinic or hospital/physician after being discharged by EMS?
7. What was their diagnosis/opinion when visiting the clinic or hospital?
8. Whas the treatment?

